\title{
ISO-RELEVANCE FUNCTIONS - A SYSTEMATIC APPROACH TO RANKING GENOMIC FEATURES BY DIFFERENTIAL EFFECT SIZE
}

\author{
SOUMYASHANT NAYAK, NICHOLAS F. LAHENS, EUN JI KIM, EMANUELA RICCIOTTI, GEORGE \\ PASCHOS, SARAH TISHKOFF, DIMITRA SARANTOPOULOU, SHAON SENGUPTA, BARRY \\ COOPERMAN, TILO GROSSER, GREGORY R. GRANT
}

\begin{abstract}
It is common to measure large numbers of features to identify those differing between experimental conditions; for example using RNA-Seq to search for differentially expressed genes. Ranking by $p$-value allows for statistical control, but has well known issues: unreliability without many replicates; and significance of biologically irrelevant effect sizes. As a result prioritization is typically performed in conjunction with effect size; the canonical one being "fold-change" $\bar{y} / \bar{x}$. However fold-change has several issues: division by zero, sensitivity to small values in the denominator, insensitivity to magnitude (1 over 2 equals 100 over 200). To mitigate these problems adding 1 to all values is a widely used heuristic; which we show using real and simulated data is typically highly sub-optimal, while the value 20 is nearly optimal in all cases. From another point of view, adding a fixed "pseudocount" to all values is essentially re-defining effect size from fold-change to something else. To explore this further, we axiomatize the concept of effect size and use this mathematical framework to study the problem in general. We also present the remarkable finding that pseudocounts strike a balance between sorting by fold-change and sorting by difference. Therefore, optimization is equivalent to finding the most harmonious balance between these two extremes. Lastly, the framework is illustrated on a fundamentally different type of problem, that of ranking di-codons by their differential abundance in the ORFeome of different species, where $p$-values are unavailable and one must solve the problem directly with effect sizes.
\end{abstract}

\section{INTRODUCTION}

A relevant example to illustrate the problem at hand is that of differential gene expression analysis using (normalized) RNA-Seq read counts, where the goal is to find the genes whose expression is "different" between two populations of samples. To do this researchers generate measurements for tens of thousands of genes in a number of replicate samples from each of the two conditions. Typically $p$-values or $q$-values are calculated for each gene and then genes are ranked by these values. In this way the genes are sorted by the power of the statistical evidence for differential expression. However genes with effect sizes too small to be biologically relevant often populate this list. A widely used heuristic approach to mitigate this problem has been to utilize volcano plots to focus on significant genes with relevant fold-changes; where "fold-change" between two values $x$ and $y$ means simply $y / x$.

Although there is copious literature on significance testing of hypotheses, there is a dearth of rigorous investigations into effect sizes, starting with even consideration of the desirable properties that a meaningful definition of effect size should have. Here we address this need with a general 
mathematical framework applicable to problems well beyond high-throughput sequencing. In the context of effect sizes based on fold-change, there have been several works that investigate the issue of its accurate estimation but do not address the question of whether fold-change itself is the most appropriate effect size to rank features in any given application. In particular, Erhard et al. use pseudocounts in an empirical Bayesian framework in order to accurately estimate the true fold-change when there are low read counts (due to random sampling involved in high-throughput sequencing). However, they are not redefining the effect size to be more appropriate to the application at hand, instead they are still just estimating fold-change, with all of its drawbacks. For example, a fold-change from 0 to 100 is equivalent to a change from a 0 to 1000 as both represent a fold-change of infinity, which may not be desirable in general.

In the fold-change between $x$ and $y$, when $x$ is zero, one typically adds some fixed positive constant, known as a "pseudocount," to both $x$ and $y$. In the literature, pseudocounts have been introduced primarily in two different ways. For one, an ad hoc pseudocount (usually 1) is used to avoid the division-by-zero issue. For another, pseudocounts have been used to define a beta prior in an empirical Bayesian procedure to estimate true fold-change. In neither of these accounts is the appropriateness of fold-change as effect size questioned.

The use of pseudocounts in ranking differential effects is perhaps one of the most widely used heuristics in biology and does mitigate the "division by zero" problem. However the division by zero problem is not the only problem nor the biggest problem with this approach. If the measurements span a wide spectrum of values, fold-change becomes problematic in how it equates ratios of different magnitudes. To illustrate this consider the following example. Suppose the data in question is RNA-Seq data and the measurements are gene level counts of reads. In this type of data counts typically range from zero to millions. Suppose we have one sample called Treatment and one sample called Control. Consider two hypothetical genes, $G_{1}$ and $G_{2}$ with the following expression counts:

\begin{tabular}{c|cc} 
Gene & Treatment & Control \\
\hline$G_{1}$ & 1000 & 2000 \\
$G_{2}$ & 1 & 2
\end{tabular}

The fold change equals two for both gene $G_{1}$ and $G_{2}$. Because of the discrete nature of RNA-Seq, we would not want to say these genes have equal differential effects. The only possible values are integer counts $0,1,2$, etc. Therefore a change of observed read-count from 1 to 2 is very likely to be due to random variation. On the other hand a change from 1000 to 2000 is stronger evidence of a real difference in population means.

Adding a pseudocount to all values, typically done to solve the division by zero problem, also mitigates this problem. But continuing with the example, if we use a pseudocount of one we would then have

\begin{tabular}{c|cc} 
Gene & Treatment & Control \\
\hline$G_{1}$ & 1001 & 2001 \\
$G_{2}$ & 2 & 3
\end{tabular}


The fold-change for $G_{2}$ went from 2 without the pseudocount, to 1.5 with, while the fold-change for $G_{1}$ went from 2 to 1.999 . However $G_{2}$ would still have the same fold change as any gene which went from 1000 to 1500 and this is still problematic. Yet virtually all studies which employ a pseudocount use a pseudocount of one or less. It is not necessary, however, to take a purely heuristic approach to this problem. Using carefully designed benchmarking data as well as real data, it is possible to perform a systematic analysis to optimize pseudocounts for certain applications. We demonstrate this for RNA-Seq below in Results.

Consider, for a moment, ranking the differential behavior of features by difference instead of foldchange. This eliminates the issue of dividing by zero; however a different problem arises, which is illustrated by the following example:

\begin{tabular}{c|cc} 
Gene & Treatment & Control \\
\hline$G_{1}$ & 10,000 & 10,100 \\
$G_{2}$ & 100 & 200
\end{tabular}

In this case both genes have a difference of 100 while we would definitely want to prioritize $G_{2}$ over $G_{1}$. This illustrates why sorting by difference is never done in practice.

We see that just as fold-change has problems with small values (and particularly with zero), difference has problems with large values. We prove below that pseudocounts provide a flexible balance between fold-change and difference allowing one to exploit the advantages and mitigate the disadvantages of both methods simultaneously.

These considerations lead naturally to a general theoretical framework that systematically describes the concept of ranking features. In order to say two features have different differential behavior from each other, we first need to develop the concept of changes being equally meaningful. This leads to the definition of "iso-relevance functions". The general framework is described first, followed by applications. The concepts are relevant to a wide range of applications. It is helpful to keep in mind the application to differential gene expression, however to demonstrate the generality of the framework another application of these methods is given, to rank di-codons by their differential usage in two species, where there are no distributions or a concept of the underlying truth and therefore no notion of statistical significance which can be used for ranking. The goal in that case is simply to prioritize features by their ability to perform a certain downstream task.

There will always be some amount of subjectivity in deciding whether two differential effects are equally relevant or not. However that's not to say it is arbitrary. Consider a gene which changes from 1000 to 2000 in RNA-Seq counts. Anybody would deem a change from 0 to 1 to be less significant. Similarly a change from 0 to 100 would probably be deemed more significant than 1000 to 2000. Somewhere in between is a grey area and we will see that the concept of pseudocount is tied implicitly and intimately with making a judgment call in this grey area. The grey area will rely on the intuition of the investigator, however the ability to systematically quantify that intuition is what is under discussion here. 


\section{Mathematical Framework}

In this section, we first illustrate how ranking by fold-change and ranking by difference are manifestations of the same ranking measure but at different scales. We state this as Main Result 1 below. After proving it, we develop the concept of iso-relevance functions as the appropriate framework for a general theory of ranking measures which yields an interpretation of the pseudocount based on a linear (complete) family of iso-relevance functions.

Main Result 1: Consider $n$-tuples, $\left(x_{1}, x_{2}, \ldots, x_{n}\right)$ and $\left(y_{1}, y_{2}, \ldots, y_{n}\right)$ representing measurements for features $G_{1}, G_{2}, \ldots, G_{n}$ under conditions $x$ and $y$ respectively. Then there is a $C>0$ such that for all $a>C$ the ordering of the features obtained using differences $x_{i}-y_{i}$, is the same as the ordering obtained using adjusted fold-changes $\frac{x_{i}+a}{y_{i}+a}$.

This follows from the following proposition and its corollary.

Proposition 2.1. Consider four positive real numbers $x_{1}, y_{1}, x_{2}, y_{2}$ such that $y_{1}-x_{1}>y_{2}-x_{2}$. There is a non-negative real number $A$ such that for all $a>A$,

$$
\frac{y_{1}+a}{x_{1}+a}>\frac{y_{2}+a}{x_{2}+a}
$$

Proof. Note that $\left(y_{1}-x_{1}\right)-\left(y_{2}-x_{2}\right)$ is a strictly positive real number. Also we have the following algebraic identity.

$$
\frac{y+a}{x+a}=\frac{(y-x)+(x+a)}{x+a}=\frac{y-x}{x+a}+1
$$

Let $a$ be a positive real number. From 2.1) it follows that

$$
\frac{y_{1}+a}{x_{1}+a}>\frac{y_{2}+a}{x_{2}+a} \Longleftrightarrow \frac{y_{1}-x_{1}}{x_{1}+a}>\frac{y_{2}-x_{2}}{x_{2}+a}
$$

Keeping in mind that $x_{1}+a, x_{2}+a$ are positive numbers, after cross-multiplication, we have that,

$$
\begin{aligned}
\frac{y_{1}-x_{1}}{x_{1}+a}>\frac{y_{2}-x_{2}}{x_{2}+a} & \Longleftrightarrow x_{2}\left(y_{1}-x_{1}\right)+a\left(y_{1}-x_{1}\right)>x_{1}\left(y_{2}-x_{2}\right)+a\left(y_{2}-x_{2}\right) \\
& \Longleftrightarrow a\left(\left(y_{1}-x_{1}\right)-\left(y_{2}-x_{2}\right)\right)>x_{1} y_{2}-x_{2} y_{1} \\
& \Longleftrightarrow a>\frac{x_{1} y_{2}-x_{2} y_{1}}{\left(y_{1}-x_{1}\right)-\left(y_{2}-x_{2}\right)}
\end{aligned}
$$

Thus we have shown

$$
\frac{y_{1}+a}{x_{1}+a}>\frac{y_{2}+a}{x_{2}+a} \Longleftrightarrow a>\frac{x_{1} y_{2}-x_{2} y_{1}}{\left(y_{1}-x_{1}\right)-\left(y_{2}-x_{2}\right)}
$$

Thus the proposition is proved by setting

$$
A=\max \left\{0, \frac{x_{1} y_{2}-x_{2} y_{1}}{\left(y_{1}-x_{1}\right)-\left(y_{2}-x_{2}\right)}\right\}
$$


Corollary 2.2. Let $\left(x_{1}, x_{2}, \ldots, x_{n}\right)$ and $\left(y_{1}, y_{2}, \ldots, y_{n}\right)$ be $n$-tuples of positive real numbers such that $y_{1}-x_{1}>y_{2}-x_{2}>\cdots>y_{n}-x_{n}$. There is a non-negative real number $A$ such that for all $a>A$,

$$
\frac{y_{1}+a}{x_{1}+a}>\frac{y_{2}+a}{x_{2}+a}>\cdots>\frac{y_{n}+a}{x_{n}+a} .
$$

Proof. Let

$$
A_{i}=\frac{x_{i} \cdot y_{i+1}-x_{i+1} \cdot y_{i}}{\left(y_{i}-x_{i}\right)-\left(y_{i+1}-x_{i+1}\right)}, \quad i \in\{1,2, \ldots, n-1\}
$$

By the previous proposition, for $a>A_{i}$, we have that,

$$
\frac{y_{i}+a}{x_{i}+a}>\frac{y_{i+1}+a}{x_{i+1}+a} .
$$

Thus if we define

$$
A=\max _{1 \leq i \leq n-1} A_{i}
$$

then for all $a>A$ it follows that

$$
\frac{y_{1}+a}{x_{1}+a}>\frac{y_{2}+a}{x_{2}+a}>\cdots>\frac{y_{n}+a}{x_{n}+a} .
$$

Let $\mathbb{R}_{+}$denote the positive real line $\{x \in \mathbb{R} \mid x \geq 0\}$. Suppose we have $n$ features $f_{1}, \ldots, f_{n}$ and measurements $x\left(f_{i}\right) \in \mathbb{R}_{+}$and $y\left(f_{i}\right) \in \mathbb{R}_{+}$for each $i=1, \ldots, n$. In light of the above results we see that the sorting features by the ratio

$$
\frac{y\left(f_{i}\right)+a}{x\left(f_{i}\right)+a}
$$

is the same as sorting by fold-change $y\left(f_{i}\right) / x\left(f_{i}\right)$ when $a=0$ and is the same as sorting by difference $y\left(f_{i}\right)-x\left(f_{i}\right)$ when $a$ is sufficiently large $(a \gg 0)$. Let $C$ be the infimum of all values with the property that sorting using a pseudocount of $a>C$ is equivalent to sorting by difference. Suppose $0<a<C$, then sorting by 2.2 with this value of $a$ strikes a balance between fold-change and difference. The closer $a$ is to zero the more weight fold-change has in this balance, and the closer $a$ is to $C$ the more weight difference has in this balance. Once $a>C$ then difference becomes $100 \%$ of the balance.

Each particular application, e.g. a specific RNA-Seq differential expression analysis, has corresponding values of $a$ that strike the balance between fold change and difference that achieves the most optimal ranking for the application. Such optimization can be done with benchmark data, when available. If we can find a range of values of $a$ that always tend to be more optimal than another range, this information can then be useful in practice. For example, suppose we find that any value of $a$ in the interval $[10,30]$ is almost always better than any value of $a$ in the interval $[0,5]$ then we can use this information to give some guidance. There's no reason to expect this to be possible a priori however as will be shown below, in RNA-Seq analysis the optimal value of $a$ appears to be remarkably stable at around $a=20$, a value considerably higher than has been typically used in practice. 
In the rest of this section, we develop the appropriate mathematical framework of iso-relevance functions to study ranking measures. For a family of linear iso-relevance functions, we will see that the ranking measure corresponds to using fold-change with a pseudocount.

Definition 2.3. We say that a function $f: \mathbb{R}_{+} \rightarrow \mathbb{R}_{+}$is an iso-relevance function if the change $x \rightarrow f(x)$ is (deemed) equally relevant for all $x$ in $\mathbb{R}_{+}$.

A straightforward example of an iso-relevance function is $f^{(0)}(x)=x$ which stands for no change.

Definition 2.4. Let $\Omega:=\left\{(x, y) \in \mathbb{R}^{2} \mid x \in \mathbb{R}_{+}, \quad y \geq x\right\} \subset \mathbb{R}_{+} \times \mathbb{R}_{+}$. We refer to $\Omega$ as the relevance octant of $\mathbb{R}^{2}$.

2.1. What properties should an iso-relevance function $f\left(\neq f^{(0)}\right)$ have? One iso-relevance function should correspond to one effect size, e.g. two-fold change. We note some properties one may reasonably expect of such a function; followed by justifications.

P0. $f(0) \neq 0$,

P1. $f>f^{(0)}$,

P2. $f-f^{(0)}$ is strictly increasing,

P3. $f / f^{(0)}$ is strictly decreasing; $\lim _{x \rightarrow \infty} \frac{f(x)}{x}$ exists and is strictly greater than 1 .

P4. $f$ is continuous.

Rationales for the above properties follow.

P0. Since $0 \rightarrow 0$ is no change, the only iso-relevance function $f$ which satisfies $f(0)=0$ is given by $f^{(0)}$ which corresponds to the null change $x \rightarrow x$.

$\mathrm{P} 1$. In this framework, we compare relevance of changes in the same direction (comparing magnitude of changes in different directions can be done by just ignoring the directionality). As $\left(f-f^{(0)}\right)(0)=f(0) \in \mathbb{R}_{+}$is strictly positive, $f(x)-f^{(0)}(x)$ must be strictly positive for all $x$, otherwise the directionality of the change is different between 0 and some value of $x \neq 0$, contradicting our assertion of equal relevance for the change at all points in $\mathbb{R}_{+}$. Also the change $x \rightarrow f(x)$ should be equally relevant to the change $0 \rightarrow f(0)$, and there cannot be multiple choices for $f(x)$.

P2. Consider the change $0 \rightarrow 20$. This change should be considered more relevant than the change $100 \rightarrow 120$ which in turn should be considered more relevant than the change $200 \rightarrow 220$, etc. The same absolute difference (20 in this case) becomes less convincing at higher values. In other words, the change $x \rightarrow x+c$ becomes less relevant as $x$ increases where $c>0$ is fixed. Thus for an iso-relevance function $f, f(x)-x$ must increase as $x$ increases. In particular, $f-f^{(0)}$ should be strictly increasing.

P3. In a similar vein as P2, we would consider the change $100 \rightarrow 125$ as less relevant than the change $500 \rightarrow 625$ which in turn would be considered less relevant than the change $1000 \rightarrow 1250$, etc. The same relative change ( $25 \%$ in this case) becomes more convincing at higher values. Thus for an iso-significance function $f, f / f^{(0)}$ should be strictly decreasing and since it is bounded below by $1, \lim _{x \rightarrow \infty} \frac{f(x)}{x}$ exists. Further we do not want the behavior 


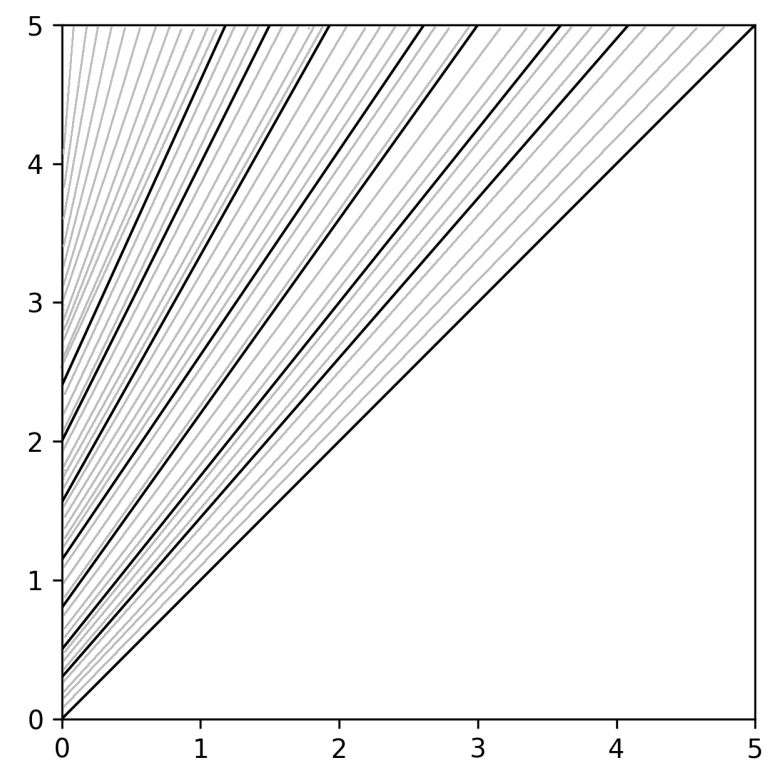

Figure 1. A linear family of iso-relevance functions

of $f\left(\neq f^{(0)}\right)$ to be similar to $f^{(0)}$ near 'infinity' (at large values) which is why we require that $\lim _{x \rightarrow \infty} \frac{f(x)}{x}$ be strictly greater than 1 .

P4. Small changes in $x$ should lead to small changes in $f(x)$.

The above defines an iso-relevance function corresponding to a particular level of effect size. We now define the notion of a complete family of iso-relevance functions corresponding to a continuum of effect sizes. It is with this family that we can rank all features in a differential massively parallel experiment with respect to each other, so that the higher a feature is on the list, the more differential the effect is for that feature. Here by 'more differential' we mean more biologically relevant rather than more statistically significant. In the context of differential gene expression, "relevant" means "differentially expressed."

As above, we state the properties first and give rationales below. Essentially a family of isorelevance functions is a partition of the relevance octant into disjoint curves with certain properties (see Fig. 1 for an example).

\subsection{What properties should a complete family $\mathcal{F}$ of iso-relevance functions have?}

P5. (uniqueness) The graphs of two distinct iso-relevance functions $f, g \in \mathcal{F}$ should not intersect. In other words, $f(x) \neq g(x)$ for any $x \in \mathbb{R}_{+}$unless $f=g$. In particular, if $f, g \in \mathcal{F}$ such that $f(0)=g(0)$, then $f=g$.

P6. (existence) For each $a>0$, there is a (unique) iso-relevance function $f_{a} \in \mathcal{F}$ such that $f_{a}(0)=a$.

P7. (completeness) For any point $(x, y)$ in the relevance octant, there is a unique iso-relevance function $f \in \mathcal{F}$ such that $y=f(x)$.

P8. (domination) If $f(0)>g(0)$, then $f(x)>g(x)$ for all $x$ in $\mathbb{R}_{+}$, 
P9. (regularity) For a fixed $x \in \mathbb{R}_{+}, f_{a}(x)$ is continuous as a function of $a$. In contrast, P4 states that $f_{a}(x)$ is continuous as a function of $x$ for fixed $a$,

P10. (closure under composition) If $f, g \in \mathcal{F}$, then $g \circ f \in \mathcal{F}$.

Rationale for the above properties follow.

P5. If the graphs of $f, g$ did intersect, we would be assigning two different effect sizes at the point of intersection (which is absurd).

P6. For any $x \in \mathbb{R}_{+}$, the change $0 \rightarrow a$ is more relevant than the change $x \rightarrow x$ but less relevant than the change $x \rightarrow M$, for $M \gg 0$. Thus there should be some intermediate value $x^{\prime}$ between $x$ and $M$ such that $0 \rightarrow a$ is equally relevant to $x \rightarrow x^{\prime}$. Defining $f_{a}(x)=x^{\prime}$, we obtain the desired iso-relevance function.

P7. For $y>x>0$, the change $0 \rightarrow 0$ is less relevant than the change $x \rightarrow y$, and the change $0 \rightarrow y$ is more relevant than the change $x \rightarrow y$. Thus there should be a number $p$ between 0 and $y$ such that the change $0 \rightarrow p$ is equally relevant to the change $x \rightarrow y$. Hence, considering the iso-relevance function $f$ which satisfies $f(0)=p$, it must be that $y=f(x)$. The uniqueness follows from property P5. Thus the graphs of the iso-relevance functions form a partition of the relevance octant (into disjoint curves).

P8. If $f(0)>g(0)$, the change $0 \rightarrow f(0)$ is more relevant than the change $0 \rightarrow g(0)$. Thus the change $x \rightarrow f(x)$ (which is equally relevant to the change $0 \rightarrow f(0)$ ) is more relevant than $x \rightarrow g(x)$ (which is equally relevant to the change $0 \rightarrow g(0)$ ).

P9. For a fixed $x \in \mathbb{R}_{+}$, small changes in $a$ should translate to small changes in $f_{a}(x)$.

P10. Let $f, g \in \mathcal{F}$. For $x, y$ in $\mathbb{R}_{+}$, by definition of iso-relevance functions, the change $x \rightarrow$ $f(x)$ is equally relevant to the change $y \rightarrow f(y)$. Similarly the change $f(x) \rightarrow g(f(x))$ is equally relevant to the change $f(y) \rightarrow g(f(y))$. Thus the change $x \rightarrow g(f(x))$ (viewed as $x \rightarrow f(x) \rightarrow g(f(x)))$ should be equally relevant to the change $y \rightarrow g(f(y))$ (viewed as $y \rightarrow f(y) \rightarrow g(f(y))$. Thus $g \circ f$ is also an iso-relevance function.

Remark 2.5. If $f \in \mathcal{F}$, then by repeated application of property $\mathrm{P} 10$ one may note that $f^{(n)}=$ $f \circ \cdots \circ f(f$ composed with itself $n$ times, $n \in \mathbb{N})$ is also in $\mathcal{F}$.

Proposition 2.6. A complete family of iso-relevance functions forms a semigroup parametrized by $\mathbb{R}_{+}$, with the identity element given by $f^{(0)}$.

Proof. From Remark 2.5, if $f$ is an iso-relevance function in $\mathcal{F}$, then so is $f^{(n)}$ for $n \in \mathbb{N}$. Note that $0<f(0)<f(f(0))$ as $f$ is strictly increasing (and $f(0)>0$ from property $\mathrm{P} 1$ ). Consider the restriction of $F$, defined in property $\mathrm{P} 9$, to the diagonal of $\mathbb{R}_{+} \times \mathbb{R}_{+}$. The real-valued function $h$ on $\mathbb{R}_{+}$, defined by $h(x)=F(x, x)$ is a strictly increasing continuous function as $F$ is continuous (property P7). As $h(0)=F(0,0)=0$ and $h(f(0))=F(f(0), f(0))=f(f(0))>f(0)$, by the intermediate value theorem, we have that $h(a)=F(a, a)=f(0)$ for some $a$ in $(0, f(0))$. Let $g$ be the iso-relevance function such that $g(0)=a$. Thus $F(g(0), g(0))=g(g(0))=f(0)$. As seen before, $g \circ g$ is also an iso-relevance function and $(g \circ g)(0)=f(0)$, which implies that $g \circ g=f$. We 
denote $g$ by $f^{(1 / 2)}$. Repeating the process, we obtain iso-relevance functions of the form $f^{\left(1 / 2^{n}\right)}$. By composition of these functions, we obtain the iso-relevance functions which can be expressed as (compositional) dyadic powers of $f$.

Note that $\left\{f^{\left(1 / 2^{n}\right)}(0)\right\}_{n=1}^{\infty}$ is a strictly decreasing sequence bounded below by 0 . Thus it has a limit which we denote by $\gamma$. Let $\phi$ be an iso-relevance function such that $\phi(0)=\gamma$. For $n$ in $\mathbb{N}$, we have that $\phi<f^{\left(1 / 2^{n}\right)}$ (property $\mathrm{P} 6$ ). Note that

$$
\lim _{x \rightarrow \infty} \frac{f(x)}{x}=\lim _{x \rightarrow \infty} \frac{f^{(1 / 2)}\left(f^{(1 / 2)}(x)\right)}{f^{(1 / 2)}(x)} \frac{f^{(1 / 2)}(x)}{x}=\lim _{x \rightarrow \infty}\left(\frac{f^{(1 / 2)}(x)}{x}\right)^{2} .
$$

Thus

$$
\lim _{x \rightarrow \infty} \frac{f^{(1 / 2)}(x)}{x}=\left(\lim _{x \rightarrow \infty} \frac{f(x)}{x}\right)^{1 / 2} .
$$

Inductively we can prove that

$$
\lim _{x \rightarrow \infty} \frac{f^{\left(1 / 2^{n}\right)}(x)}{x}=\left(\lim _{x \rightarrow \infty} \frac{f(x)}{x}\right)^{1 / 2^{n}}, \forall n \in \mathbb{N} .
$$

Hence,

$$
1 \leq \lim _{x \rightarrow \infty} \frac{\phi(x)}{x} \leq\left(\lim _{x \rightarrow \infty} \frac{f(x)}{x}\right)^{1 / 2^{n}}, \forall n \in \mathbb{N}
$$

and we conclude that

$$
\lim _{x \rightarrow \infty} \frac{\phi(x)}{x}=1
$$

From property P3, we have that $\phi=f^{(0)}$ and thus $\lim _{n \rightarrow \infty} f^{\left(1 / 2^{n}\right)}(0)=\gamma=0$.

As dyadic numbers are dense in the reals, using the above conclusion and a routine continuity argument, we can define $f^{(\alpha)}$ for all positive real numbers $\alpha$ such that for $\alpha, \beta \in \mathbb{R}_{+}, f^{(\alpha)} \circ f^{(\beta)}=$ $f^{(\alpha+\beta)}$. Moreover for any $a \in \mathbb{R}_{+}$there is an $\alpha$ in $\mathbb{R}_{+}$such that $f^{(\alpha)}(0)=a$ and $f^{(\alpha)}$ is the unique iso-relevance function attaining the value $a$ at 0 .

Corollary 2.7. A non-trivial iso-relevance function $f\left(\neq f^{(0)}\right)$ belongs to precisely one complete family of iso-relevance functions. In other words, a single non-trivial element generates the whole family.

Definition 2.8. In Proposition 2.6, we noted that one non-trivial iso-relevance function in $\mathcal{F}$ is enough to obtain the rest of the functions in $\mathcal{F}$. When generating a complete family of iso-relevance functions from one function, we call the generator used the seed.

We first explore the consequences of using a linear seed to generate a complete family of isosignificance functions.

Example 2.9. Let $a>0, b>1$. We will see below that the iso-relevance function $f^{(1)}(x)=a+b x$ is a seed for a complete family of iso-relevance functions. (Note that if $a=0$, we recover the usual fold-change but this does not constitute a complete family of iso-significance functions since they all intersect at the origin.) One may interpret the seed in the following manner: the change 0 to 
$a$ is deemed equally relevant to the relative change $x \rightarrow b x$ in the limit as $x \rightarrow \infty$. The other iso-relevance functions in the corresponding complete family are given by

$$
f^{(\alpha)}(x)=a\left(\frac{b^{\alpha}-1}{b-1}\right)+b^{\alpha} x, \quad \alpha \in \mathbb{R}_{+} .
$$

In other words, the change $0 \rightarrow a\left(\frac{b^{\alpha}-1}{b-1}\right)$ should be deemed equally relevant to the change $x \rightarrow b^{\alpha} x$ for larger numbers $x$.

In order to see that (2.3) defines a complete family of iso-relevance functions, we only need check properties P7, P8 and P10 as the rest of the properties are straightforward to verify.

Proof of P7. Let $(x, y)$ be a point in the relevance octant. Note that

$$
\begin{aligned}
y=f^{(\alpha)}(x)=a\left(\frac{b^{\alpha}-1}{b-1}\right)+b^{\alpha} x & \Longleftrightarrow y+\frac{a}{b-1}=b^{\alpha}\left(x+\frac{a}{b-1}\right) \\
& \Longleftrightarrow \alpha=\frac{\ln \left(y+\frac{a}{b-1}\right)-\ln \left(x+\frac{a}{b-1}\right)}{\ln b} .
\end{aligned}
$$

As $y \geq x$, we have $\alpha \geq 0$ and thus there is a unique iso-relevance function $f^{(\alpha)}$ such that $y=f^{(\alpha)}(x)$. Proof of P8. Note that $f^{(\alpha)}(0)>f^{(\beta)}(0) \Rightarrow b^{\alpha}>b^{\beta} \Rightarrow \alpha>\beta \Rightarrow f^{(\alpha)}(x)>f^{(\beta)}(x)$ for all $x \in \mathbb{R}_{+}$. Proof of P10. For $\alpha, \beta \in \mathbb{R}_{+}$, and $x$ in $\mathbb{R}_{+}$, we have

$$
\begin{aligned}
\left(f^{(\alpha)} \circ f^{(\beta)}\right)(x)=f^{(\alpha)}\left(f^{(\beta)}(x)\right) & =a\left(\frac{b^{\alpha}-1}{b-1}\right)+b^{\alpha} f^{(\beta)}(x) \\
& =a\left(\frac{b^{\alpha}-1}{b-1}\right)+b^{\alpha} \cdot\left(a\left(\frac{b^{\beta}-1}{b-1}\right)+b^{\beta} x\right) \\
& =a\left(\frac{b^{\alpha}-1}{b-1}\right)+a\left(\frac{b^{\alpha+\beta}-b^{\alpha}}{b-1}\right)+b^{\alpha+\beta} x \\
& =a\left(\frac{b^{\alpha+\beta}-1}{b-1}\right)+b^{\alpha+\beta} x=f^{(\alpha+\beta)}(x) .
\end{aligned}
$$

Thus $f^{(\alpha)} \circ f^{(\beta)}$ is in the family of functions defined.

In Fig. 1, the iso-relevance functions depicted are with respect to the seed given by $2+2 x$.

Definition 2.10. Let $\mathcal{F}$ be a complete family of iso-relevance functions with a given seed $f=f^{(1)}$. For $(x, y)$ in the relevance octant, let $\alpha \in \mathbb{R}_{+}$be such that $y=f^{(\alpha)}(x)$. Then $\alpha$ is called the relevance level of the change $x \rightarrow y$ (with respect to $\mathcal{F}$ ) which we take as the corresponding effect size.

We state the result in the proof of property P7 in Example 2.9 as a proposition below.

Proposition 2.11. Let $(x, y)$ be a point in the relevance octant and consider the complete isorelevance family generated by $a+b x$ where $a>0, b>1$. Then the relevance level of the change $x \rightarrow y$ is given by

$$
\alpha=\frac{\ln \left(y+\frac{a}{b-1}\right)-\ln \left(x+\frac{a}{b-1}\right)}{\ln b} .
$$


Remark 2.12. The above proposition may be interpreted in the following manner. The change $x_{1} \rightarrow y_{1}$ is more relevant than the change $x_{2} \rightarrow y_{2}$ if

$$
\frac{\ln \left(y_{1}+\frac{a}{b-1}\right)-\ln \left(x_{1}+\frac{a}{b-1}\right)}{\ln b}>\frac{\ln \left(y_{2}+\frac{a}{b-1}\right)-\ln \left(x_{2}+\frac{a}{b-1}\right)}{\ln b},
$$

which is equivalent to

$$
\frac{y_{1}+\frac{a}{b-1}}{x_{1}+\frac{a}{b-1}}>\frac{y_{2}+\frac{a}{b-1}}{x_{2}+\frac{a}{b-1}} .
$$

Main Result 2: If we choose $b=2$ and let $a$ be such that the change $0 \rightarrow a$ is as significant as the change $x \rightarrow 2 x$ (two-fold change) in the limit as $x \rightarrow \infty$, then $\frac{y+a}{x+a}$ is a measure of the relevance level of the change. This observation provides a philosophical interpretation of the pseudocount value as the change from 0 that is deemed as relevant as two-fold change in the limit.

What we have shown above is that pseudocounts naturally arise from the consideration of a linear seed in our framework.

\section{Results}

We will discuss two fundamentally different applications of the main conclusions drawn in the previous section. In the second example, there is no alternative to ranking by effect size as the problem does not involve anything probabilistic.

3.1. RNA-Seq. The mathematical framework of iso-relevance functions suggests that one should not treat the pseudocount as an ad hoc solution to the division-by-zero issue. This often leads researchers to be conservative in their choice of pseudocount, the most common values being 1 or less.

Datasets: An investigation into optimal pseudocounts in RNA-Seq differential expresssion (DE) analysis was first performed based on twenty RNA-Seq benchmark datasets (each with two experimental conditions and eight replicates per condition). These were obtained from transformations of real data, from several different organisms and tissues from several different labs and institutions:

- Human Blood ( $\approx 9$ million reads per sample on average)

- Human UHRR ( $\approx 9.5$ million reads per sample on average)

- Mouse Liver ( $\approx 21$ million reads per sample on average)

- Mouse Aorta ( $\approx 37$ million reads per sample on average)

- Arabidopsis ( $\approx 23$ million reads per sample on average)

Details on how the benchmarking data was generated are given in Materials and Methods. Essentially these are real data with all the properties of real data known and unknown, with some number of differentially expressed genes created by random subsampling of reads of select genes. Each dataset has two conditions with eight replicates each. In half of the datasets, 200 genes out of the complete set of genes for the species under consideration are differentially expressed and in the other half 1000 genes out of the complete set of genes are differentially expressed. There are roughly $30 \mathrm{~K}$ genes that are not differential. These two types of datasets are further divided into 
two subtypes each; Balanced datasets (B) have half DE genes upregulated and the other half downregulated, and unbalanced datasets (U) have all genes upregulated. See Table 1 for a summary of the 20 datasets.

\begin{tabular}{|c|c|c|c|c|}
\cline { 2 - 5 } \multicolumn{1}{c|}{} & \multicolumn{2}{c|}{1000 DE genes } & \multicolumn{2}{c|}{200 DE genes } \\
\hline Arabidopsis & Balanced & Unbalanced & Balanced & Unbalanced \\
\hline Human Blood & Balanced & Unbalanced & Balanced & Unbalanced \\
\hline Mouse Liver & Balanced & Unbalanced & Balanced & Unbalanced \\
\hline Mouse Aorta & Balanced & Unbalanced & Balanced & Unbalanced \\
\hline UHRR & Balanced & Unbalanced & Balanced & Unbalanced \\
\hline
\end{tabular}

TABLE 1. Description of the 20 RNA-Seq datasets for benchmarking.

There are two main classes of 'effect size' we are interested in for a comparative study:

(i) $\frac{y+c}{x+c}(c>0)$, where $y$ is the average of the gene's expression values over the eight replicates in the first group, and $x$ is the average of the gene's expression values over the eight replicates in the second group.

(ii) PsiLFC (see Erhard [7]) estimate for fold-change.

For a given notion of effect size, we may sort the genes in terms of that effect size. For the dataset with 200 (1000, respectively) DE genes, we are interested in how many of the top 200 (1000, respectively) genes according to this sorting are true positives. For each dataset, a graph was therefore prepared which plots the pseudocount $c$ on the horizontal axis against the size of the intersection of the top 200 (1000, respectively) genes in this list with the known list of DE genes. Further, on each graph, the number of true positives determined by PsiLFC (Erhard ) is indicated by a horizontal dashed line.

The results for the $200 \mathrm{DE}$ genes datasets (1000 DE genes datasets, respectively) are shown in Fig. 2 (Fig. 3 , respectively). A surprising consistency is observed in the optimal value of $c$ across datasets.

\section{Observations:}

1. In all cases the maximal intersection with the truth occurs around $c=20$. In fact anything from $c=15$ to $c=25$ is close to optimal.

2. The number of DE genes had little effect on the optimal value of $c$.

3. The widely used value $c=1$ is extremely sub-optimal in all cases.

4. Performance is also extremely sub-optimal for large values of $c$ demonstrating the intuitive contention that ranking by difference is also a bad idea.

5. In almost all cases, the true positive rate for PsiLFC underperformed compared to adjusted fold-change with a pseudocount of 20. Only in the Mouse Liver unbalanced datasets did PsiLFC perform comparably to this approach. 

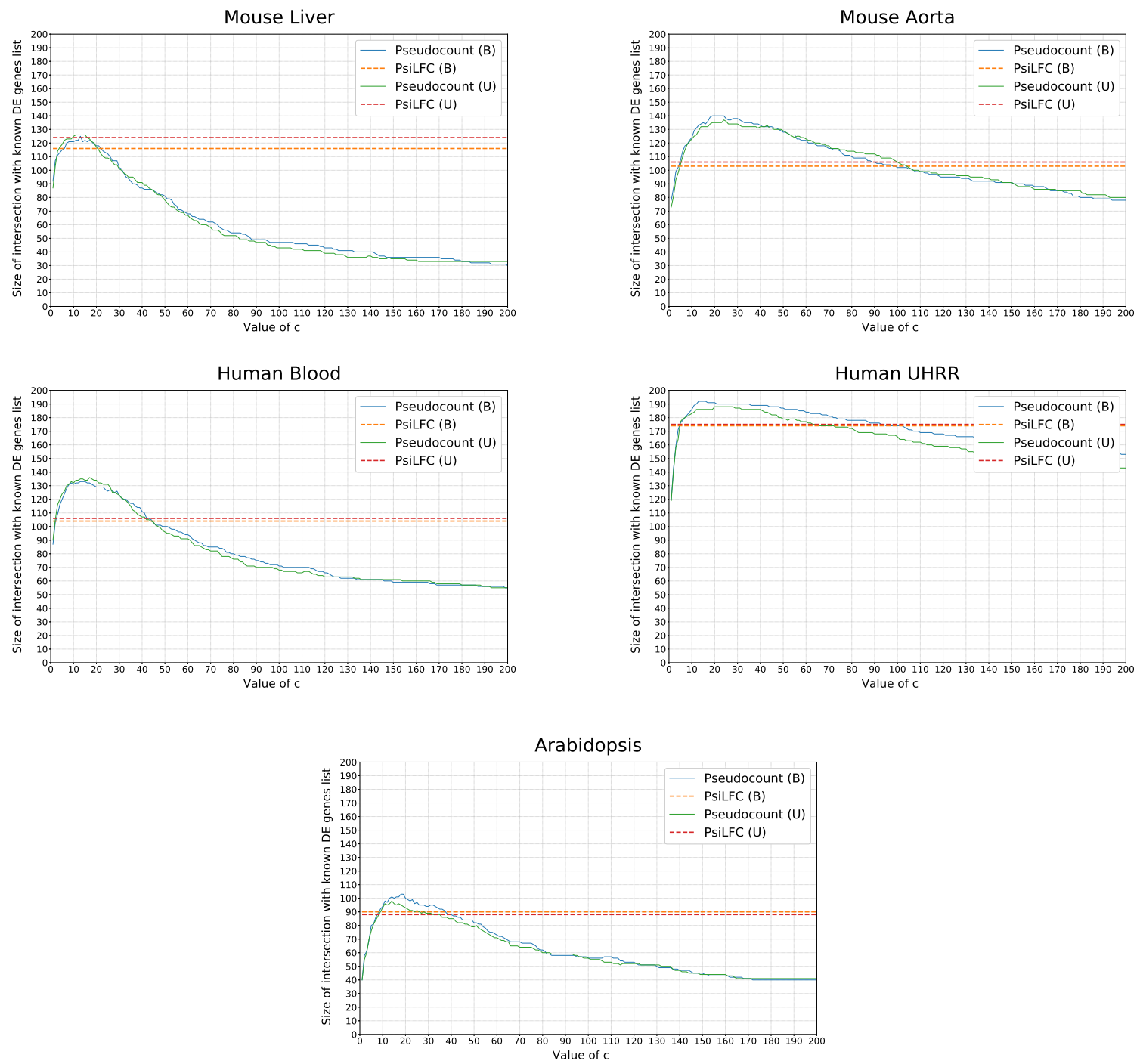

FiguRE 2. Results for datasets with 200 DE genes. (B) - Balanced, (U) - Unbalanced.

6. In all cases, the true positive rate for PsiLFC is better compared to adjusted fold-change with pseudocount of 1 .

The above results were based on eight replicates in each condition. It is important to explore the optimal value of $c$ also with fewer replicates (down to as few as one). The results for the 200 DE genes mouse aorta dataset (1000 DE genes dataset, respectively) are shown in Fig. 4 (Fig. 5. respectively).

\section{Observations:}

1. Once again, $c=1$ is much too small. Greatly improved performance is obtained by increasing $c$ from 1 to 10 . Once again, surprisingly, $c=20$ is close to optimal in all data sets. 
bioRxiv preprint doi: https://doi.org/10.1101/381814; this version posted February 5, 2019. The copyright holder for this preprint (which was not certified by peer review) is the author/funder, who has granted bioRxiv a license to display the preprint in perpetuity. It is made available under aCC-BY 4.0 International license.
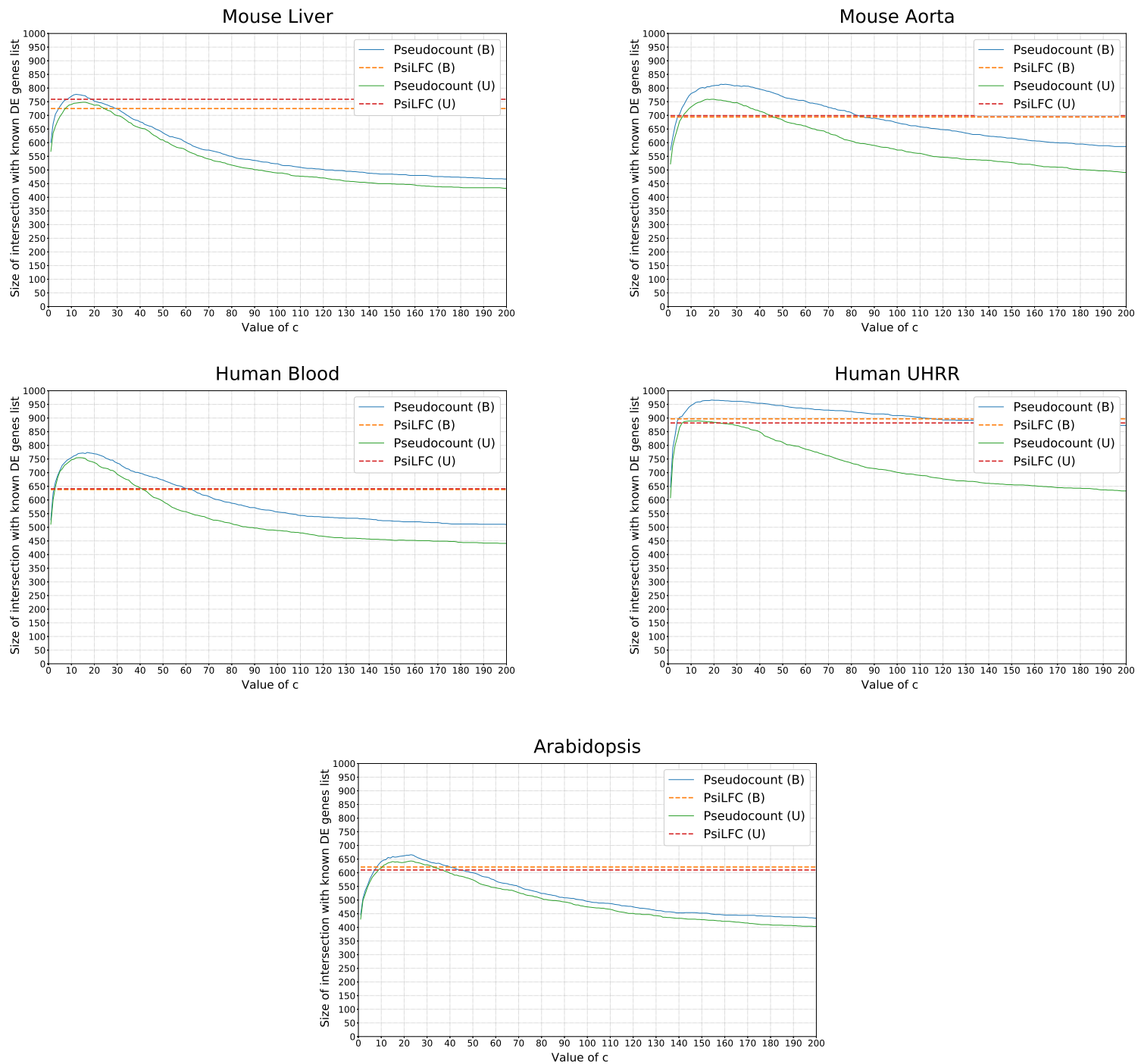

FiguRE 3. Results for datasets with 1000 DE genes. (B) - Balanced, (U) - Unbalanced.

2. As can be seen from the figures (Fig. 6, Fig. 7), the smaller the number of replicates the bigger the improvement by using $c=20$ instead of $c=1$.

3. Once again, PsiLFC underperformed compared to adjusted fold-change with pseudocount of 20 (see Table 3.)

4. PsiLFC performed better compared to adjusted fold-change with pseudocount of 1 .

3.1.1. Application to Pathway Enrichment Analysis. Another standard way to compare two differential expression analyses is by which analysis leads to more meaningful and powerful pathway enrichment $p$-values. We investigated the sensitivity of the pathway enrichment $p$-values on the value of the pseudocount in an RNA-Seq dataset (GEO - GSE117029). The purpose of the experiment for which the data was generated was to determine whether there is a differential effect on the transcriptome of mice based on time of influenza infection. In order to identify the relevant list 
bioRxiv preprint doi: https://doi.org/10.1101/381814; this version posted February 5, 2019. The copyright holder for this preprint (which was not certified by peer review) is the author/funder, who has granted bioRxiv a license to display the preprint in perpetuity. It is made available under aCC-BY 4.0 International license.
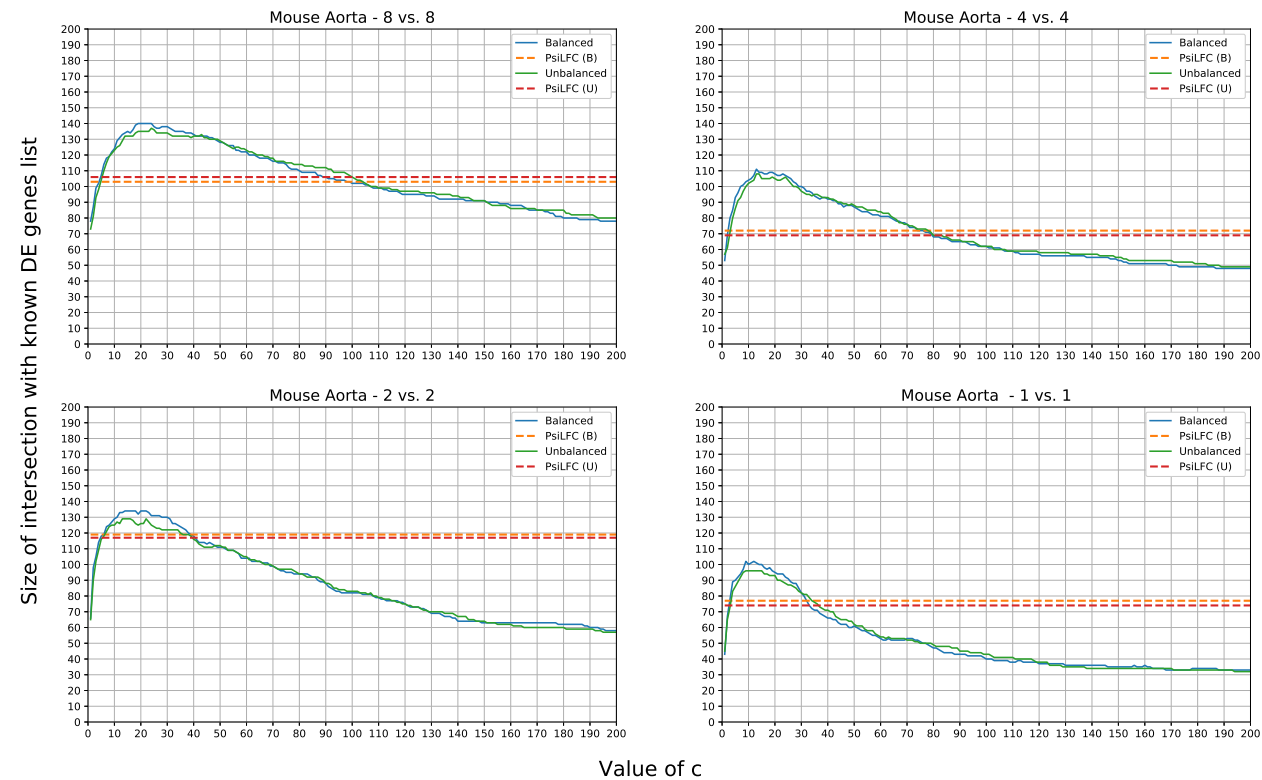

Value of $c$

FiguRE 4. Effect of number of replicates in 200 DE genes dataset (mouse liver). (B) - Balanced, (U) - Unbalanced.
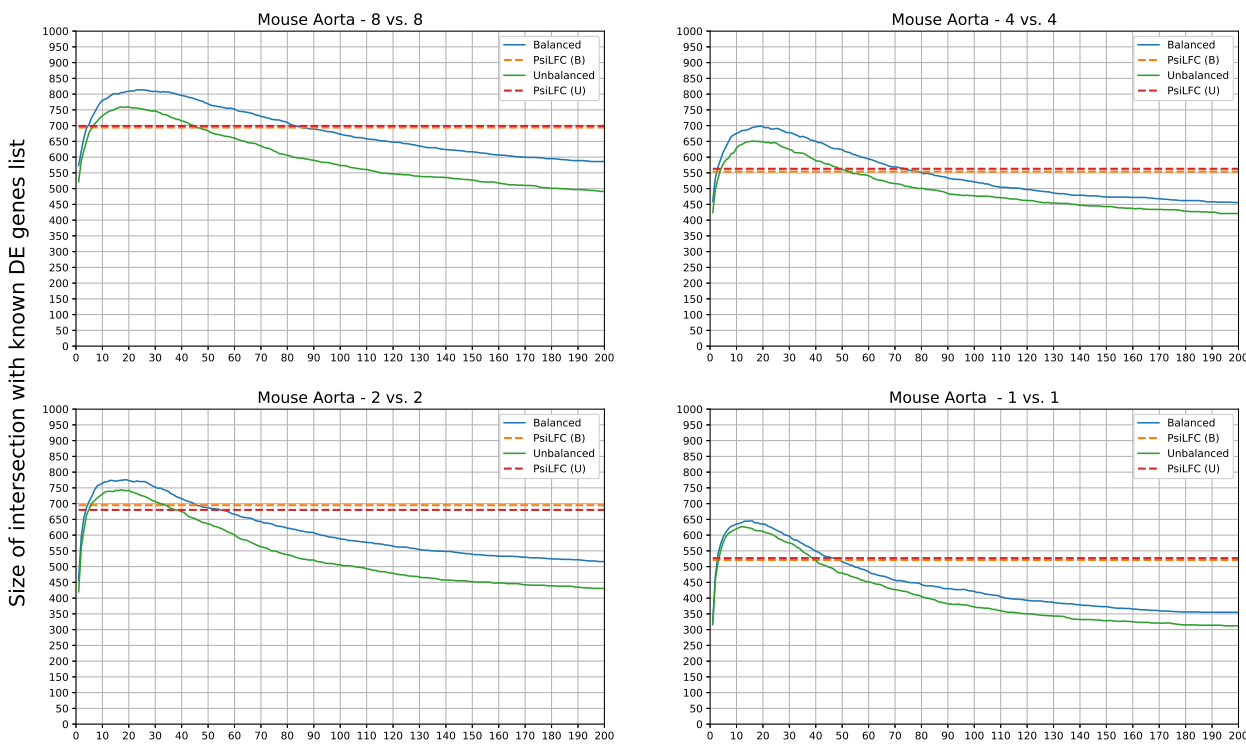

Value of $c$

FiguRE 5. Effect of number of replicates in 1000 DE genes dataset (mouse liver). (B) - Balanced, (U) - Unbalanced. 
bioRxiv preprint doi: https://doi.org/10.1101/381814; this version posted February 5,2019 . The copyright holder for this preprint (which was not certified by peer review) is the author/funder, who has granted bioRxiv a license to display the preprint in perpetuity. It is made available under aCC-BY 4.0 International license.

\begin{tabular}{|c|c|c|c|c|c|}
\hline & & \multicolumn{4}{|c|}{ True positive rate } \\
\hline & & \multicolumn{2}{|c|}{$1000 \mathrm{DE}$ genes } & \multicolumn{2}{|c|}{200 DE genes } \\
\hline & & Balanced & Unbalanced & Balanced & Unbalanced \\
\hline \multirow{3}{*}{ Arabidopsis } & $\mathrm{FC}$, pseudocount $=1$ & $44.6 \%$ & $43.0 \%$ & $20.0 \%$ & $20.0 \%$ \\
\hline & FC, pseudocount $=20$ & $66.2 \%$ & $63.8 \%$ & $50.0 \%$ & $46.5 \% s$ \\
\hline & PsiLFC & $62.1 \%$ & $61.0 \%$ & $45.0 \%$ & $44.0 \%$ \\
\hline \multirow{3}{*}{ Human Blood } & FC, pseudocount $=1$ & $52.7 \%$ & $51.1 \%$ & $43.5 \%$ & $45.0 \%$ \\
\hline & FC, pseudocount $=20$ & $76.9 \%$ & $73.7 \%$ & $64.5 \%$ & $67.0 \%$ \\
\hline & PsiLFC & $63.8 \%$ & $64.1 \%$ & $52.0 \%$ & $53.0 \%$ \\
\hline \multirow{3}{*}{ Mouse Liver } & FC, pseudocount $=1$ & $60.1 \%$ & $56.8 \%$ & $46.0 \%$ & $43.5 \%$ \\
\hline & FC, pseudocount $=20$ & $75.2 \%$ & $73.7 \%$ & $59.0 \%$ & $58.5 \%$ \\
\hline & PsiLFC & $72.5 \%$ & $75.9 \%$ & $58.0 \%$ & $62.0 \%$ \\
\hline \multirow{3}{*}{ Mouse Aorta } & FC, pseudocount $=1$ & $57.3 \%$ & $52.2 \%$ & $39.0 \%$ & $36.5 \%$ \\
\hline & FC, pseudocount $=20$ & $80.9 \%$ & $75.9 \%$ & $70.0 \%$ & $68.5 \%$ \\
\hline & PsiLFC & $69.4 \%$ & $69.9 \%$ & $51.5 \%$ & $53 \%$ \\
\hline \multirow{3}{*}{ UHRR } & FC, pseudocount $=1$ & $64.7 \%$ & $60.8 \%$ & $60.0 \%$ & $59.5 \%$ \\
\hline & FC, pseudocount $=20$ & $96.5 \%$ & $88.4 \%$ & $95.5 \%$ & $94.0 \%$ \\
\hline & PsiLFC & $89.7 \%$ & $88.2 \%$ & $87.0 \%$ & $87.5 \%$ \\
\hline
\end{tabular}

TABLE 2. True positive rate of the various ranking methods on the 20 datasets.

The best true positive rate for a particular dataset is in bold.

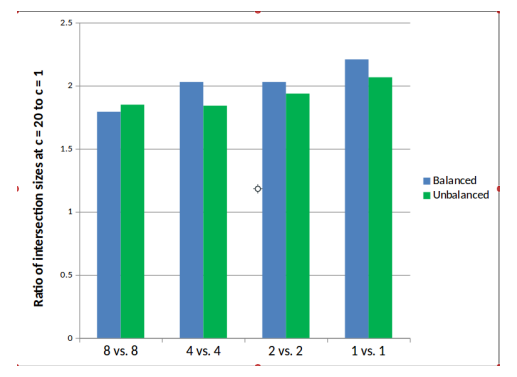

FiguRE 6. Comparison across number of replicates (200 DE genes dataset)

of genes in terms of their differential change in gene expression across the time-points, we employed four methods; two-way ANOVA $p$-values, DESeq2 two factor analysis $p$-values (using unnormalized read counts for the samples), differential change in adjusted fold-change with pseudocount of 1 , and with pseudocount of 20 . The details of the methods may be found in the supplementary material. The results of the pathway enrichment analysis performed using IPA are shown in Table 4.

Observations : 


\begin{tabular}{|c|c|c|c|c|c|}
\hline & \multicolumn{4}{|c|}{ True positive rate (Mouse Aorta datasets) } \\
\hline & & \multicolumn{2}{|c|}{1000 DE genes } & \multicolumn{2}{|c|}{200 DE genes } \\
\hline & & Balanced & Unbalanced & Balanced & Unbalanced \\
\hline \multirow{3}{*}{8 vs. 8} & FC, pseudocount $=1$ & $57.3 \%$ & $52.2 \%$ & $39.0 \%$ & $36.5 \%$ \\
\hline & FC, pseudocount $=20$ & $80.9 \%$ & $75.9 \%$ & $70.0 \%$ & $68.5 \%$ \\
\hline & PsiLFC & $69.4 \%$ & $69.9 \%$ & $51.5 \%$ & $53 \%$ \\
\hline \multirow{3}{*}{4 vs. 4} & FC, pseudocount $=1$ & $45.9 \%$ & $42.4 \%$ & $26.5 \%$ & $28.5 \%$ \\
\hline & FC, pseudocount $=20$ & $69.7 \%$ & $64.9 \%$ & $54.0 \%$ & $52.5 \%$ \\
\hline & PsiLFC & $55.4 \%$ & $56.3 \%$ & $36.0 \%$ & $34.5 \%$ \\
\hline \multirow{3}{*}{2 vs. 2} & FC, pseudocount $=1$ & $45.8 \%$ & $42.0 \%$ & $33.0 \%$ & $32.5 \%$ \\
\hline & FC, pseudocount $=20$ & $77.3 \%$ & $74.2 \%$ & $67.0 \%$ & $63.0 \%$ \\
\hline & PsiLFC & $69.5 \%$ & $68.0 \%$ & $59.5 \%$ & $58.5 \%$ \\
\hline \multirow{3}{*}{1 vs. 1} & FC, pseudocount $=1$ & $32.3 \%$ & $31.6 \%$ & $21.5 \%$ & $22.5 \%$ \\
\hline & FC, pseudocount $=20$ & $63.5 \%$ & $61.2 \%$ & $47.5 \%$ & $46.5 \%$ \\
\hline & PsiLFC & $52.1 \%$ & $52.7 \%$ & $38.5 \%$ & $37.0 \%$ \\
\hline
\end{tabular}

TABLE 3. True positive rate of the various ranking methods for the mouse aorta datasets with varying number of replicates. The best true positive rate for a particular dataset is in bold.

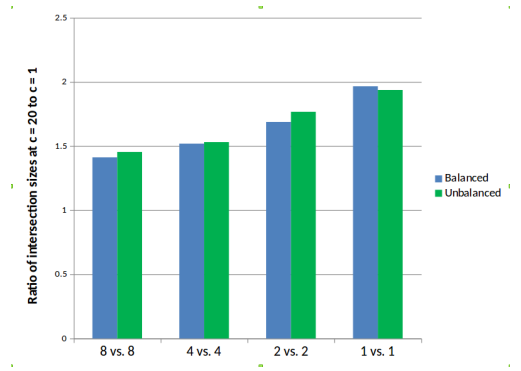

FiguRE 7. Comparison across number of replicates (1000 DE genes dataset)

1. The enrichment $p$-values are generally significantly lower in the gene list obtained from adjusted FC with pseudocount as compared to the list obtained by using two-way ANOVA interaction $p$-values or DESeq2 two-factor analysis interaction $p$-values.

2. The enrichment $p$-values are generally lower in the case of adjusted FC with pseudocount of 20 compared to adjusted FC with pseudocount of 1 . For example, in the case of the pathway "Role of Pattern Recognition Receptors in Recognition of Bacteria and Viruses" which is highly relevant to the biology of the experiment, the improvement elevates the pathway from being of marginal interest to being quite significant.

3. There are exceptions to the above two observations. For example, the pathway "Activation of IRF by Cytosolic Pattern Recognition Receptors" has significantly better enrichment 


\begin{tabular}{|c|c|c|c|c|}
\hline \multirow[b]{2}{*}{$\begin{array}{l}\text { Ingenuity Canonical Path- } \\
\text { ways (IPA) }\end{array}$} & \multicolumn{4}{|c|}{$-\log p$-value } \\
\hline & $\begin{array}{l}\text { 2-Way } \\
\text { ANOVA } \\
\text { interaction }\end{array}$ & $\begin{array}{l}\text { DESeq2 } \\
\text { Two-Factor } \\
\text { interaction }\end{array}$ & $\begin{array}{l}\text { DC list with } \\
\text { pseudocount } \\
=1\end{array}$ & $\begin{array}{l}\text { DC list with } \\
\text { pseudocount } \\
=20\end{array}$ \\
\hline $\begin{array}{l}\text { Granulocyte Adhesion and } \\
\text { Diapedesis }\end{array}$ & 4.16 & $9.52^{\dagger}$ & $14.1^{\dagger}$ & $17.2^{\dagger}$ \\
\hline $\begin{array}{l}\text { Agranulocyte Adhesion and } \\
\text { Diapedesis }\end{array}$ & 3.32 & 5.82 & 12.4 & 13.6 \\
\hline $\begin{array}{l}\text { Role of Pattern Recognition } \\
\text { Receptors in Recognition of } \\
\text { Bacteria and Viruses }\end{array}$ & 5.11 & 5.31 & 5.93 & 10.7 \\
\hline $\begin{array}{l}\text { Role of Hypercytokine- } \\
\text { mia/hyperchemokinemia in } \\
\text { the Pathogenesis of Influenza }\end{array}$ & not significant & 3.26 & 11.2 & 9.66 \\
\hline $\begin{array}{l}\text { Communication between In- } \\
\text { nate and Adaptive Immune } \\
\text { Cells }\end{array}$ & 3.62 & 3.62 & 5.84 & 8.42 \\
\hline Nicotine Degradation II & not significant & not significant & 4.48 & 8.1 \\
\hline $\begin{array}{l}\text { Differential Regulation of Cy- } \\
\text { tokine Production in Intesti- } \\
\text { nal Epithelial Cells by } I L-17 A \\
\text { and } I L-17 F\end{array}$ & not significant & not significant & 7.58 & 7.84 \\
\hline $\begin{array}{l}\text { Activation of IRF by Cytoso- } \\
\text { lic Pattern Recognition Re- } \\
\text { ceptors }\end{array}$ & $7.21^{\dagger}$ & 3.16 & 3.55 & 4 \\
\hline
\end{tabular}

TABLE 4. Comparison of pathway enrichment $p$-values based on top 900 genes from the four ranking procedures.

$\dagger$ represents $p$-value for the topmost enriched pathway obtained from the ranking procedure.

$p$-values in the list obtained from 2-way ANOVA $p$-values. This could partly be because we use the same number of DE genes (900) for each list and the better enriched pathways tend to have better gene coverage reducing the gene coverage for the less enriched pathways.

4. The DESeq2 two-factor analysis is slightly more informative than two-way ANOVA but less informative than differential change analysis from FC using pseudocounts.

In the Discussion section (see $\$ 4$, we discuss possible reasons as to why the use of effect size to rank genes for the eventual pathway enrichment analysis is a better idea compared to the use of statistical significance for ranking. 
3.2. Differential Dicodon Usage. We now turn to a fundamentally different problem where $p$ values are not available. The frequency of neighboring amino acids in the proteome varies from species to species. For example valine and arginine are very rarely adjacent in human, however they are very often adjacent in Zika virus. This information can potentially be exploited as a diagnostic because the active translation of any particular di-codon can be detected with FRET signaling using fluorescent tRNAs (Barhoom et al). Towards this goal it is necessary to identify the tRNA-tRNA pairs that have the best chance of providing a sufficiently informative signature to reveal the presence of the parasite infecting a human cell - in other words, identifying pairs that are high in the virus proteome and low in the human proteome. As using all 1035 possible pairs is not practical with the current state of the technology, we seek a small handful that provide sufficient discrimination. Therefore we need to rank di-codons by the differential frequencies of the occurrence of tRNA-tRNA anticodon pairs in translating the two proteomes.

As the relative frequencies of most of the tRNA-tRNA pairs are close to 0 , the ranking problem based on differential relative frequencies amongst the tRNA-tRNA pairs is a challenging one. This is also a fundamentally different problem from the RNA-Seq example discussed above as there are no replicates and it is not amenable to conventional statistical analysis where benchmarking data may be generated with the known ground truth. Since there are roughly 1000 tRNA-tRNA pairs, a prior $i$ we expect the relative frequency of each tRNA-tRNA pair to be close to $0.1 \%$. By the very nature of the problem, the appropriate choice of pseudocount in this scenario cannot be directly determined. But from Corollary 2.2 and the discussion following it, a spectrum of values of pseudocount can be evaluated until satisfactory results are obtained, without having to straitjacket our choice to a value very close to zero. In this case, we appeal to the interpretation mentioned in Main Result 2 that the pseudocount represents the value $a$ such that the change $0 \rightarrow a$ is as significant as a two-fold change for large values.

The coding sequences of IVA H1N1 strain (GenBank id - GQ160811.1, Korea 2009) and IVB Victoria strain (GenBank id - CY040455.1, Malaysia 2004) downloaded from the NCBI Nucleotide database were used to obtain the relative frequencies (in percentage) of tRNA-tRNA pairs. We experimented with various values of pseudocount to sort dicodons by differential usage and visually compared the top dicodons in the list which revealed 0.5 to be an effective value of the pseudocount. Using this pseudocount of 0.5 , the adjusted fold-change was computed to rank the tRNA-tRNA pairs for their abilities to distinguish between IVA and IVB infection. In Figure 8(A)-(B), the heatmaps display the relative frequencies of the 1035 tRNA-tRNA pairs in IVA H1N1, IVB Victoria, respectively. The labels are the anticodons associated with the tRNAs. In Figure $8(\mathrm{C})-(\mathrm{D})$, for the tRNA-tRNA pairs that best distinguish IVA H1N1 and IVB Victoria (called DiDi pairs for the two viruses), the relative frequencies in IVA H1N1, IVB Victoria, respectively, are shown. In Table 5 . the relative frequencies of the top DiDi pairs are noted, three each for IVA H1N1 and IVB Victoria. 


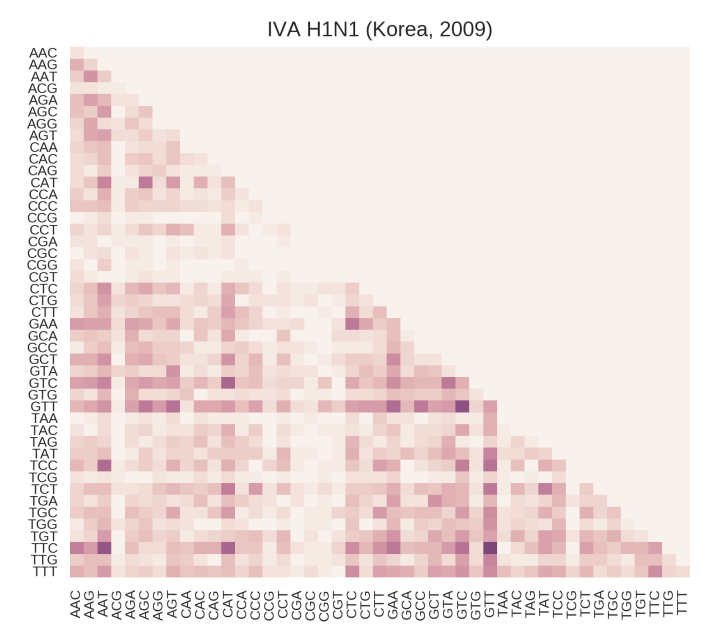

(A) IVA H1N1 tRNA-tRNA pair relative frequency (in percentage)

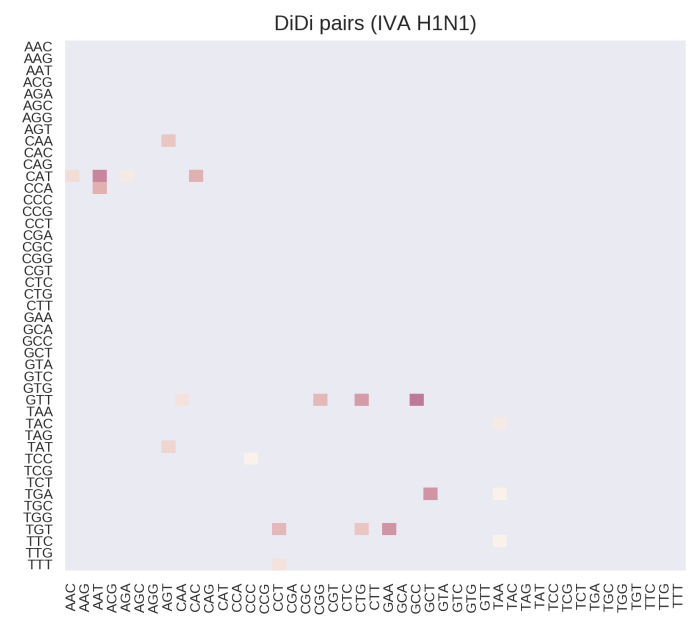

(c) DiDi pair relative frequency for IVA H1N1 (in percentage)

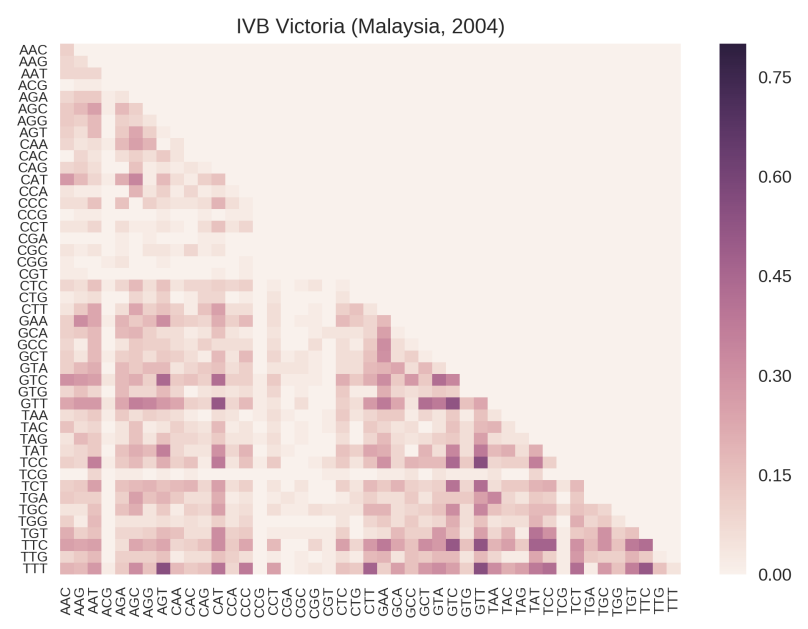

(в) IVB Victoria tRNA-tRNA pair relative frequency (in percentage)

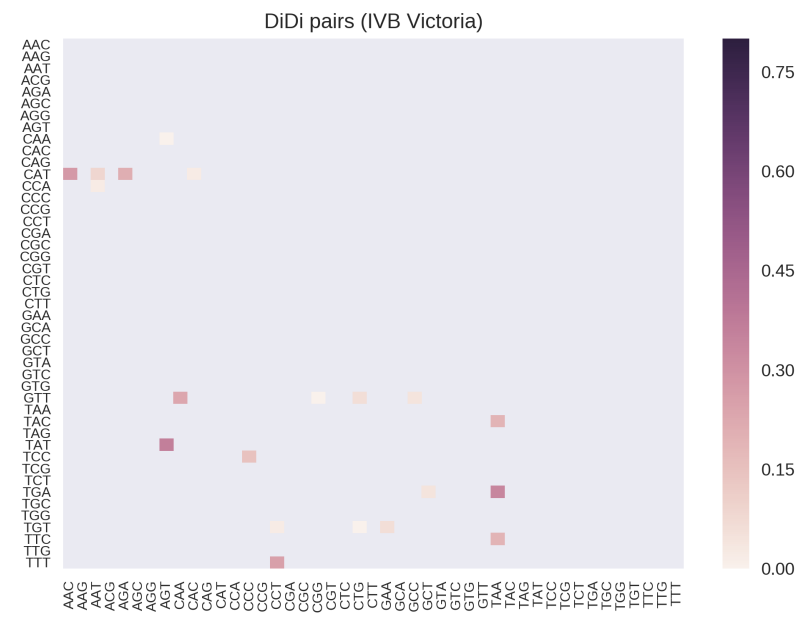

(D) DiDi pair relative frequency for IVB Victoria (in percentage)

Figure 8.

\section{Discussion}

The procedure of adding a pseudocount of one to all values arises very naturally as a solution to the division-by-zero problem, and certainly many investigators have come to this solution independently. However, it is perhaps counter-intuitive to believe that the final results would be highly sensitive to this value. Given how widely used this heuristic is, there have been surprisingly few investigations into this issue. Erhard and Zimmer [6] give an interpretation of pseudocounts, specific to RNA-Seq differential gene expression analysis, as parameters in a prior distribution, when using 


\begin{tabular}{|c|c|c|}
\cline { 2 - 3 } \multicolumn{1}{c|}{} & \multicolumn{2}{c|}{ relative frequency } \\
\hline tRNA-tRNA pair & IVA H1N1 & IVB Victoria \\
\hline TAA-TGA & 0 & 0.340 \\
\hline TAA-TTC & 0 & 0.191 \\
\hline AGA-CAT & 0.022 & 0.212 \\
\hline GCC-GTT & 0.383 & 0.042 \\
\hline CGG-GTT & 0.180 & 0 \\
\hline GCT-TGA & 0.293 & 0.042 \\
\hline
\end{tabular}

TABLE 5. Top DiDi pairs for IVA H1N1 - IVB Victoria comparison

Bayesian MAP estimation of the fold-change. However the focus is on using pseudocounts to estimate fold-change with confidence intervals rather than using them to explore alternative effect sizes that may be more appropriate for a given context. Their pseudocounts are tailored to each gene in an empirical Bayes framework to incorporate prior knowledge. The goals here are quite different. However the use of empirical Bayes is questionable regardless as it uses expression values across all genes to formulate a Bayesian model to estimate a statistic of interest (such as fold-change) and provide confidence intervals. This relies on a strong assumption of homogeneous behavior across all genes which certainly does not hold in general and can lead to misleading conclusions. Additionally there is no practical guidance on how to estimate optimal pseudocounts. Further, the results were not related to the ranking problem. The only other paper we identified which performed a systematic benchmarking of pseudocounts is Warden et al [2], however they limited the range of their pseudocount to be between 0 and 1; and as we've seen the optimal value may be much larger. Additionally this result is specific to RNA-Seq whereas our results are applicable to far more general situations. Furthermore, they did not attempt to develop a general framework in which to investigate these issues. The closest to a generalized framework that has been published is an obscure work by Aczél et al [1] which develops a framework in which to compare $p$-values. As $p$-values are between zero and one they focus on the unit square in the 1st quadrant, while an analysis of fold-change involves the entire 1st quadrant.

In Erhard et al [6], the fold-change is discussed as a means of canceling bias which is assumed to grow linearly with the feature counts. The assumption of linearity of bias is deemed more appropriate at the read sequence level during the PCR step in RNA-Seq than the same assumption in terms of read counts at the aggregate features level (such as genes, transcripts, etc.) The authors introduce a count ratio model based on local read counts aligned to a certain genomic position. The parameters used in the prior beta distribution for the log-fold-change ultimately feature in the estimator for the fold-change in the form of pseudocounts. The total fold-change of a feature is suggested to be modeled as a weighted average of local fold-changes, where positions with many reads contribute more to the total fold-change than positions with fewer reads, however, it is never actually implemented. The validation of the methods are done in E. coli using technical replicates. 
For one, this may not generalize to studies involving eukaryotes. For another, the model only addresses technical bias (such as PCR bias) and does not deal with the case of biological replicates, which is of primary interest in practice.

The results here could conceivably be generalized to ranking features by $T$-statistic, which would be advantageous in incorporating variance into effect size which fold-change ignores. Let $\mathbf{x}=$ $\left(x_{1}, \ldots, x_{n}\right), \mathbf{y}=\left(y_{1}, \ldots, y_{n}\right)$ be two vectors in $\mathbb{R}_{+}^{n}$. The $T$-statistic is given by a function $T$ : $\mathbb{R}_{+}^{n} \times \mathbb{R}_{+}^{n} \mapsto \mathbb{R}$ which sends $(\mathbf{x}, \mathbf{y})$ to $(\overline{\mathbf{x}}-\overline{\mathbf{y}}) /\left(s_{p} \sqrt{\frac{2}{n}}\right)$ where $s_{p}$ denotes the pooled standard deviation. In this case, problems arise when the variance of both groups is small. Firstly since the denominator is essentially given by these variances, that can cause the $T$-statistic to blow up just like dividing by very small numbers in the fold-change. This naturally leads to a higher dimensional analog of the problem. Instead of iso-relevance functions of one variable, addressing these issues will require generalizing this concept to $n$ dimensions. Secondly, if a hypothetical dataset consists of observations with zero variance (or all with the same variance), it essentially reduces to the one-dimensional problem of assessing relevance of change of real values. The standard procedure of sorting features according to $p$-values (from the $T$-statistic) reduces to sorting by difference which, as discussed earlier, is more convincing at smaller scales but not so much at larger scales. Thus one should keep in mind that any generalization to higher dimensions should be compatible with the concept of iso-relevance functions in a lower dimensional space. Thirdly, the use of the T-test implicitly assumes that the variance of the expression of each gene is the same. When the scale of the expression level is different, the variance may be significantly different. To address this issue, similar to using pseudocounts, Huber et al introduced what they called a "fudge factor" added to the denominator. The rankings of features can be remarkably sensitive to this parameter (see Grant et al [4]). This will be the focus of future work.

We point out another fundamental difference between the usage of ranking by fold-changes vs. $p$-values with regards to pathway enrichment analyses. The $p$-values measure our confidence in the fact that the corresponding genes are differentially expressed. They convey little information about the 'effect size' or biological relevance of the change. Although a $p$-value, or more precisely, FDR (false discovery rate) cutoff works well for determining the pool of DE genes, it is by nature quite ineffective in actually sorting the DE genes by effect size. There are two issues here which deserve further investigation. First, for the purpose of pathway enrichment analysis, the problem of effect size persists at the pathway level. Although two pathways may be similarly enriched (comparable hypergeometric $p$-values), the average effect size may be quite different. Second, often finding the right FDR cut-off for pathway enrichment analysis is an issue of trial-and-error and preliminary investigations have shown that this phenomenon may be because we do not take the effect size into account for sorting. We will leave that discussion for another occasion.

\section{Materials And Methods}

Benchmarking Data. The RNA-Seq data was obtained from the following GEO datasets:

(i) Mouse Liver (GSE115264) 
(ii) Mouse Aorta (GSE95802)

(iii) Human Blood (GSE99719)

(iv) Human UHRR (GSE47774)

(v) Arabidopsis (GSE80744),

and manipulated into benchmarking data. For each data set we started with 16 samples from two conditions. (Comment on normalization method) Samples were permuted to have two new groups with 8 samples from each condition in each group. The distribution of $p$-values was then plotted to make sure it does not deviate significantly from uniform. From this data, 200 genes were chosen from each of 10 (average) expression levels low to high. For each gene chosen, reads were randomly removed from the samples in one group to mimic differential expression. 10 different levels of differential expression were created from 1.1 fold to infinite fold. This was done in two ways, one way all 200 genes are up-regulated; in the second way half are upregulated and half are downregulated. Another set of data sets was generated similarly, however with 1000 DE genes instead of 200. Benchmarking data is described in more detail and available for download at http://bioinf .itmat. upenn.edu/benchmarking/rnaseq/port/datasets.php

In Table 6, we note the samples from the Mouse Aorta dataset used for the comparative study of adjusted fold-change with various pseudocounts and PsiLFC estimator for fold-change, with respect to varying the number of replicates.

\begin{tabular}{|c|c|c|}
\cline { 2 - 3 } \multicolumn{1}{c|}{} & Condition 1 & Condition 2 \\
\hline \multirow{3}{*}{8 vs. 8} & Sample_4138_grp1, Sample_4140_grp1, & Sample_4139_grp2, Sample_4141_grp2, \\
& Sample_4142_grp1, Sample_4144_grp1, & Sample_4143_grp2, Sample_4145_grp2, \\
& Sample_4146_grp1, Sample_4148_grp1, & Sample_4147_grp2, Sample_4149_grp2, \\
& Sample_4151_grp1, Sample_4154_grp1 & Sample_4153_grp2, Sample_4155_grp2 \\
\hline 4 vs. 4 & Sample_4138_grp1, Sample_4140_grp1, & Sample_4139_grp2, Sample_4141_grp2, \\
& Sample_4142_grp1, Sample_4144_grp1 & Sample_4143_grp2, Sample_4145_grp2 \\
\hline 2 vs. 2 & Sample_4138_grp1, Sample_4146_grp1 & Sample_4139_grp1, Sample_4147_grp1 \\
\hline 1 vs. 1 & Sample_4138_grp1 & Sample_4139_grp1 \\
\hline
\end{tabular}

TABle 6. Description of the samples chosen from Mouse Aorta dataset for the comparative analysis using varying number of replicates.

Mouse Influenza data. The RNA-Seq data was obtained from a GEO dataset (GSE117029) for an experiment involving male mice infected at two distinct time points (three replicates at 6 a.m., four replicates at 6 p.m.) with influenza virus and three replicates each from PBS mock infected controls at the same two time points. The purpose of the experiment was to determine whether there is a differential effect on the transcriptome of mice based on time of infection. As several genes are differentially expressed simply by virtue of the circadian rhythm or other timedependent effects, it was necessary to account for this in the analysis. A standard approach is to 
perform 2-way ANOVA with two categorical variables: "treatment status" (control or treated) and "time" (6am or 6pm) and to look for genes with significant interaction $p$-values. Although it is generally considered suboptimal with just three replicates per condition, for the sake of comparison we performed a two-way ANOVA analysis to find interaction effects and sorted the list of genes by the interaction p-values. We performed a similar study with a DESeq2 two-factor interaction model with the factors being time and treatment, and sorted the list of genes by the interaction $p$-values. At each timepoint the adjusted fold change of gene expression level was computed relative to the corresponding control group using a pseudocount of 20. Genes which had coefficient of variation $>3$ were filtered out and the remaining genes were then ranked based on the ratios of their adjusted fold-change at the two timepoints. Out of a total of 20401 genes, we found 900 genes exhibiting a differential log fold change (DC) of atleast 0.67 in magnitude which corresponds to about 5 -fold change in the adjusted fold-changes. We repeated the above ranking procedure for the list of genes using a pseudocount of 1 . For a consistent comparison, we noted the top 900 genes in all three of the ranking procedures to check for pathway enrichment.

Di-Codon data. The coding sequences for IVA H1N1 strain (Korea - 2009), IVB Victoria strain (Malaysia - 2004) were downloaded from the NCBI Nucleotide database using the GenBank IDs GQ160811.1, CY040455.1, respectively. The di-codon frequency in the CDS's of both viruses was computed. The final goal of this exercise, which is out of scope of the paper, was to identify DiDi pairs which have low usage frequency in the human ORFeome as that would be the background in any diagnostic procedure. As viruses use the host's translational machinery, in order use DiCoMPS ([5]) one needs to reinterpret dicodon usage in terms of tRNA-tRNA pair usage. In general there is a fairly strong correlation between relative frequency of codon usage and the number of tRNA genes having an anticodon sequence complementary to the codon. For the common 20 amino acids, there are 55 isoacceptor tRNAs that are complementary to the 61 codons. The 6 isoacceptors (GCC, AGT, ACG, AAG, GAA, GTG) that completely decode 2 codons each, have an $A$ or $G$ at the 5 '-end of the anticodon sequence that forms a base pair with either a $U$ or $C$ at the 3'-end of the codon - the wobble position. In addition there are 8 pairs of isoacceptor tRNAs (see supplementary Table 1, colored in yellow), that differ by having an $A$ or $G$ at the 5'-end of the anticodon sequence that have very different numbers of tRNA genes $(8-38$ fold differences) but are complementary to codons with similar relative frequency of codon usages (http://gtrnadb.ucsc.edu/GtRNAdb2/genomes/eukaryota/Hsapi38/Hsapi38-summary-codon.html). The clear inference is that in these cases the dominant tRNA isoacceptor will generally translate both codons. Finally, there are 2 pairs of anticodons (see supplementary Table 1, colored in blue), again differing by having an $A$ or $G$ at the 5 -end of the anticodon sequence, where the difference in the number of tRNA genes is less dramatic ( 36 -fold differences) and are complementary to codons with similar frequency of codon usages. In these cases it is likely that both tRNAs will be involved in translating the two codons, but again the dominant tRNA isoacceptor is likely to play the major role. Based on the above reasoning, we focussed on the $45(=61-(6+8+2))$ tRNA 
isoacceptors that are most strongly involved in translation. With this mapping of codons to tRNA isoacceptors, the relative frequencies of each of the $1035=45 \cdot\left(\frac{45+1}{2}\right)$ tRNA-tRNA anticodon pairs were computed.

\section{REFERENCES}

[1] J. Aczél, G. Rote, J. Schwaiger; Webs, Iteration Groups and Equivalent Changes in Probabilities , Quarterly of Applied Mathematics 54 (1996), 475-499.

[2] Warden CD, Yuan YC, Wu X.; Optimal Calculation of RNA-Seq Fold-Change Values, International Journal of Computational Bioinformatics and In Silico Modeling 2(6), (2013), 285-292.

[3] Tusher V, Tibshirani R, Chu C; Significance analysis of microarrays applied to transcriptional responses to ionizing radiation, Proceedings of the National Academy of Sciences 98:51165121 (2001).

[4] Grant GR, Liu J, Stoeckert C (2005); A practical false discovery rate approach to identifying patterns of differential expression in microarray data

[5] Barhoom S, Farrell I, Shai B, Dahary D, Cooperman BS, Smilansky Z, Elroy-Stein O, Ehrlich M.; Dicodon monitoring of protein synthesis (DiCoMPS) reveals levels of synthesis of a viral protein in single cells, Nucleic Acids Res. 2013 Oct;41(18):e177.

[6] Erhard F, Zimmer R.; Count ratio model reveals bias affecting NGS fold changes, Nucleic Acids Res. 2015 Vol. 43. No.20.

[7] Erhard F.; Estimating pseudocounts and fold changes for digital expression measurements, Bioinformatics, bty471, https://doi.org/10.1093/bioinformatics/bty471

[8] Michael Love, Wolfgang Huber, Simon Anders; Moderated estimation of fold change and dispersion for RNA-seq data with DESeq2, Genome Biology (2014) 15:550 DOI 10.1186/s13059-014-0550-8

itmat, Perelman School of Medicine, University of Pennsylvania, Philadelphia, PA 19104

E-mail address: nsoum@upenn.edu

$U R L:$ https://nsoum.github.io 